\title{
VISCOUS SPLITTING FOR THE UNBOUNDED PROBLEM OF THE NAVIER-STOKES EQUATIONS
}

\author{
LUNG-AN YING
}

\begin{abstract}
The viscous splitting for the exterior initial-boundary value problems of the Navier-Stokes equations is considered. It is proved that the approximate solutions are uniformly bounded in the space $L^{\infty}\left(0, T ; H^{s+1}(\Omega)\right)$, $s<\frac{3}{2}$, and converge with a rate of $O(k)$ in the space $L^{\infty}\left(0, T ; H^{1}(\Omega)\right)$, where $k$ is the length of the time steps.
\end{abstract}

\section{INTRODUCTION}

Let $\Omega$ be a domain in the space $\mathbb{R}^{2}$. An initial-boundary value problem of the Navier-Stokes equation is given as

$$
\begin{gathered}
\frac{\partial u}{\partial t}+(u \cdot \nabla) u+\frac{1}{\rho} \nabla p=\nu \Delta u+f, \quad x \in \Omega, t>0 \\
\nabla \cdot u=0, \quad x \in \Omega, t>0, \\
\left.u\right|_{x \in \partial \Omega}=0, \\
\left.u\right|_{t=0}=u_{0}(x) .
\end{gathered}
$$

If $\Omega=\mathbb{R}^{2}$, then the boundary condition (1.3) disappears and the problem reduces to a pure initial value problem.

Beale and Majda [4] proved the convergence of a viscous splitting scheme for the initial value problem, where equation (1.1) was split in each time step into an Euler equation and a linear Stokes equation. This scheme was related to the vortex method [6], a numerical approach for high Reynold's number flow. Therefore, it is interesting to consider not only pure initial value problems, but also initial-boundary value problems. It is known that there is a boundary layer near the boundary, and that vortices are created and turbulence may develop. From the point of view of numerical analysis, the boundary condition for the Euler equation is different from that of the Navier-Stokes equation; the changing of the boundary condition in each time step creates singularities of the approximate solutions.

Alessandrini, Douglis, and Fabes considered the viscous splitting of the initial-boundary value problem in bounded domains [3], where the solutions of

Received January 9, 1989; revised August 1, 1989.

1980 Mathematics Subject Classification (1985 Revision). Primary 35Q10, 76D05. 
the Euler equation were replaced by polynomials. Convergence was proved, but it is not known whether this scheme is numerically realizable. Benfatto and Pulvirenti proved the convergence of a scheme for the initial-boundary value problem in the half plane [5]. A distribution vortex sheet, whose support is just the boundary, was inserted as in the vortex method, and a Neumann condition for the vorticity was introduced to replace the velocity boundary condition. The combination of those two steps generated an approximate no-slip condition at the boundary.

The author of this paper considered this problem in bounded domains [13][17]; a correction step was applied to maintain the no-slip condition too, but this operator was bounded in $H^{s+1}, s \geq 0$, the velocity boundary condition for the diffusion step was exact, and a nonhomogeneous term was added to the Stokes equation to neutralize the error of the above correction step. Convergence was proved. Numerical results have been obtained which will appear in a separate paper.

The purpose of this paper is to study this problem for unbounded domains. For simplicity we assume that the boundary $\partial \Omega$ of $\Omega$ is sufficiently smooth, simply closed, and $\Omega$ is its exterior. We also assume that flows tend to zero at infinity. The problem of the physically interesting case of flows having uniform velocity at infinity is still open. A simplification of the proof would suffice for the bounded case.

We now briefly summarize our main results. Denote by $x=\left(x_{1}, x_{2}\right)$ or $y=\left(y_{1}, y_{2}\right)$ a point in $\mathbb{R}^{2}$. The usual notations $H^{s}(\Omega)$ and $W^{m, p}(\Omega)$ for Sobolev spaces, and $\|\cdot\|_{s}$ and $\|\cdot\|_{m, p}$ for norms, are used throughout this paper. For the problems $(1.1)-(1.4)$, we assume that $\nabla \cdot u_{0}=0, u_{0} \in\left(H_{0}^{1}(\Omega)\right)^{2} \cap$ $\left(H^{3}(\Omega)\right)^{2}, f \in L^{\infty}\left(0, T ;\left(H^{3}(\Omega)\right)^{2}\right) \cap W^{1, \infty}\left(0, T ;\left(H^{1}(\Omega)\right)^{2}\right)$, and the solution $u \in L^{\infty}\left(0, T ;\left(H^{4}(\Omega)\right)^{2}\right) \cap W^{1, \infty}\left(0, T ;\left(H^{5 / 2}(\Omega)\right)^{2}\right)$, where $T$ is a positive constant.

We construct a projection operator

$\Theta:\left\{u \in\left(H^{1}(\Omega)\right)^{2} ; \nabla \cdot u=0,(u \cdot n, 1)_{\partial \Omega}=0\right\} \rightarrow\left\{u \in\left(H_{0}^{1}(\Omega)\right)^{2} ; \nabla \cdot u=0\right\}$,

such that

$$
\|\Theta u\|_{s+1} \leq C\|u\|_{s+1} \quad \forall s \geq 0
$$

where $n$ is the unit outward normal vector, $(\cdot, \cdot)_{\partial \Omega}$ is the inner product of $L^{2}(\partial \Omega)$, and $C$ is a constant depending on $s$. We will give an example of $\Theta$ in $\S 2$.

The following scheme is considered: We divide the interval $[0, T]$ into equal subintervals with length $k$. Then we construct $\tilde{u}_{k}(t), \tilde{p}_{k}(t), u_{k}(t), p_{k}(t)$ on each interval $[i k,(i+1) k), i=0,1, \ldots$, according to the following procedure. 
In the first step, we solve the following problem on the interval $[i k,(i+1) k)$ :

$$
\begin{gathered}
\frac{\partial \tilde{u}_{k}}{\partial t}+\left(\tilde{u}_{k} \cdot \nabla\right) \tilde{u}_{k}+\frac{1}{\rho} \nabla \tilde{p}_{k}=f, \\
\nabla \cdot \tilde{u}_{k}=0, \\
\left.\tilde{u}_{k} \cdot n\right|_{x \in \partial \Omega}=0, \\
\tilde{u}_{k}(i k)=u_{k}(i k-0),
\end{gathered}
$$

where $u_{k}(-0)=u_{0}$.

In the second step-the projection-we construct $\Theta \tilde{u}_{k}((i+1) k-0)$.

In the third step, we solve the following problem on the interval $[i k,(i+1) k)$ :

$$
\begin{gathered}
\frac{\partial u_{k}}{\partial t}+\frac{1}{\rho} \nabla p_{k}=\nu \Delta u_{k}+\frac{1}{k}(I-\Theta) \tilde{u}_{k}((i+1) k-0), \\
\nabla \cdot u_{k}=0, \\
\left.u_{k}\right|_{x \in \partial \Omega}=0, \\
u_{k}(i k)=\Theta \tilde{u}_{k}((i+1) k-0),
\end{gathered}
$$

where $I$ is the identity operator. In these formulas the spacial variable $x$ is suppressed for simplicity.

Our main result is the following:

Theorem. If $u$ is the solution of problem (1.1)-(1.4), $\tilde{u}_{k}, u_{k}$ the solutions of problems (1.6)-(1.13), and if $0 \leq s<\frac{3}{2}$, then there is a constant $k_{0}>0$ such that

$$
\begin{gathered}
\sup _{0 \leq t \leq T}\left(\left\|u_{k}(t)\right\|_{s+1},\left\|\tilde{u}_{k}(t)\right\|_{s+1}\right) \leq M, \\
\sup _{0 \leq t \leq T}\left(\left\|u(t)-u_{k}(t)\right\|_{1},\left\|u(t)-\tilde{u}_{k}(t)\right\|_{1}\right) \leq M^{\prime} k
\end{gathered}
$$

for $0<k \leq k_{0}$, where the constants $k_{0}, M, M^{\prime}$ depend only on the domain $\Omega$, the constants $\nu, s, T$, the operator $\Theta$, the functions $f, u_{0}$, and the solution $u$ of $(1.1)-(1.4)$.

The existence and uniqueness of the solution $u_{k}$ is known [9, Chapter 4 , Theorem 1 and §2], and using an argument similar to [12], we can get the existence and uniqueness of $\tilde{u}_{k}$; the regularity of $u_{k}, \tilde{u}_{k}$ is also obtained. Although the existence in [12] is merely local, we will show that the step length is independent of $i$.

\section{Preliminaries}

In this paper we always denote by $C$ a generic constant which depends only on the domain $\Omega$, the operator $\Theta$, and the constants $\nu, s, T$; by $C_{0}$ a generic constant which depends only on the domain $\Omega$, the operator $\Theta$, the constants $\nu, s, T$, the known functions $f, u_{0}$, and the solution $u$ of (1.1)(1.4); by $C_{1}, C_{2}, \ldots, M_{0}, M_{1}, \ldots$ some other constants which are determined according to special requirements. 
Let $E^{0}(\Omega)$ be a subset of $L^{2}(\Omega)$ such that $\omega \in E^{0}(\Omega)$ if and only if $\omega \in$ $L^{2}(\Omega)$ and there is a $u \in\left(L^{2}(\Omega)\right)^{2}$ such that $\omega=-\nabla \Lambda u$, where $\nabla \Lambda=$ $\left(\partial_{2},-\partial_{1}\right), \partial_{i}=\partial / \partial x_{i}$. We define a norm

$$
[\varphi]_{1}=\left(\int_{\Omega}|\nabla \varphi|^{2} d x\right)^{1 / 2}
$$

in $C_{0}^{\infty}(\bar{\Omega})$. Let $E^{1}(\Omega)$ be the closure of $C_{0}^{\infty}(\bar{\Omega})$ with respect to the norm $[\cdot]_{1}$, and let $E_{0}^{1}(\Omega)$ correspond to $C_{0}^{\infty}(\Omega)$. Letting $\omega \in E^{0}(\Omega)$, we consider the boundary value problem

$$
\left\{\begin{array}{l}
-\Delta \varphi=\omega \\
\left.\varphi\right|_{\partial \Omega}=0
\end{array}\right.
$$

The weak statement of $(2.1)$ is: find $\varphi \in E_{0}^{1}(\Omega)$ such that

$$
(\nabla \Lambda \varphi, \nabla \Lambda \psi)=(\omega, \psi)=(u, \nabla \Lambda \psi) \quad \forall \psi \in E_{0}^{1}(\Omega),
$$

where $\omega=-\nabla \Lambda u$. It is easy to see that

$$
(\nabla \Lambda \varphi, \nabla \Lambda \varphi)=[\varphi]_{1}^{2} .
$$

By the Lax-Milgram theorem, (2.2) possesses a unique solution. Setting $\psi=\varphi$ in $(2.2)$, we get $[\varphi]_{1}^{2} \leq\|u\|_{0}[\varphi]_{1}$, hence

$$
[\varphi]_{1} \leq\|u\|_{0} .
$$

If $\omega \in E^{0}(\Omega)$ and $\varphi$ is the solution of (2.2), we define a norm

$$
[\omega]_{0}=\left(\|\omega\|_{0}^{2}+[\varphi]_{1}^{2}\right)^{1 / 2}
$$

in $E^{0}(\Omega)$. It is easy to see that $E^{0}(\Omega)$ is a Hilbert space.

Let $D^{m}$ be a differential operator of $m$ th order, $m \geq 0, D^{m}=\partial^{m} / \partial x_{1}^{i} \partial x_{2}^{j}$, $i+j=m$. We assume that $\omega \in E^{0}(\Omega) \cap H^{m}(\Omega)$; then by the regularity of the solutions of elliptic equations [2], we have for the solution $\varphi$ of (2.2) that $\varphi \in H_{\mathrm{loc}}^{m+\hat{2}}(\Omega)$. From (2.2) we get

$$
\left(\nabla \Lambda \varphi, \nabla \Lambda D^{m} \partial_{i} \psi\right)=\left(\omega, D^{m} \partial_{i} \psi\right) \quad \forall \psi \in C_{0}^{\infty}(\Omega) .
$$

Integrating by parts, we obtain

$$
\left(\nabla \Lambda D^{m} \partial_{i} \varphi, \nabla \Lambda \psi\right)=-\left(D^{m} \omega, \partial_{i} \psi\right) .
$$

We first assume $\omega \in C_{0}^{\infty}(\bar{\Omega})$; then $\varphi$ is the solution of the Laplace equation near infinity. From the expansion of $\varphi$ at infinity it is easy to see that $\nabla \Lambda D^{m} \partial_{i} \varphi \in L^{2}(\Omega)$, hence (2.4) also holds for $\psi \in E_{0}^{1}(\Omega)$.

Let trace $b=\left.D^{m} \partial_{i} \varphi\right|_{\partial \Omega}$; then [1, Theorem 7.53]

$$
\|b\|_{1 / 2, \partial \Omega} \leq C\|\varphi\|_{m+2, \Omega^{\prime}},
$$

where $\Omega^{\prime}$ is a neighborhood of $\partial \Omega$. By the Poincare inequality and the local estimate of the solution $\varphi$,

$$
\|\varphi\|_{m+2, \Omega^{\prime}} \leq C\left(\|\omega\|_{m}+[\varphi]_{1}\right) .
$$


Let $D^{m} \partial_{i} \varphi=\varphi_{1}+\varphi_{2}$, where $\varphi_{1} \in E_{0}^{1}(\Omega)$ is the solution of

$$
\left(\nabla \Lambda \varphi_{1}, \nabla \Lambda \psi\right)=-\left(D^{m} \omega, \partial_{i} \psi\right) \quad \forall \psi \in E_{0}^{1}(\Omega),
$$

and $\varphi_{2} \in E^{1}(\Omega)$ is the solution of

$$
\begin{aligned}
\left(\nabla \Lambda \varphi_{2}, \nabla \Lambda \psi\right) & =0 \quad \forall \psi \in E_{0}^{1}(\Omega), \\
\left.\varphi_{2}\right|_{\partial \Omega} & =b .
\end{aligned}
$$

$\varphi_{2}$ is a bounded harmonic function, hence $\left\|\nabla \varphi_{2}\right\|_{0} \leq C\|b\|_{1 / 2, \partial \Omega}$. By (2.3), $\left[\varphi_{1}\right]_{1} \leq\left\|D^{m} \omega\right\|_{0}$. We have

$$
\left[D^{m} \partial_{i} \varphi\right]_{1} \leq C\left(\|\omega\|_{m}+[\varphi]_{1}\right) \leq C\left(\|\omega\|_{m}+[\omega]_{0}\right) .
$$

$C_{0}^{\infty}(\bar{\Omega})$ is dense in $E^{0}(\Omega)$; therefore $(2.5)$ holds for all $\omega \in E^{0}(\Omega) \cap H^{m}(\Omega)$. We define $[\varphi]_{m}=\|\nabla \varphi\|_{m-1}$ for $m \geq 1$; then

$$
[\nabla \varphi]_{m+1} \leq C\left(\|\omega\|_{m}+[\omega]_{0}\right) .
$$

By the interpolation theorem [10, Chapter 1, Theorem 5.1], we have

$$
[\nabla \varphi]_{s+1} \leq C\left(\|\omega\|_{s}+[\omega]_{0}\right) \quad \forall s \geq 0 .
$$

We denote by $E_{0}^{m}(\Omega)$ the closure of $C_{0}^{\infty}(\Omega)$ with respect to the norm $[\cdot]_{m}$.

Now we give an example of an operator $\Theta$ which satisfies inequality (1.5). Construct $\chi \in C_{0}^{\infty}(\bar{\Omega})$ such that $\chi \equiv 1$ near the boundary $\partial \Omega$. Let $\Omega^{\prime}$ be a bounded domain whose boundary consists of simply closed curves $\Gamma$ and $\partial \Omega$, where $\Gamma$ is outside of $\partial \Omega$, sufficiently smooth, and $\operatorname{supp} \chi \subset \bar{\Omega}^{\prime}$. Let $\varphi$ be a stream function of $u$ in $\Omega^{\prime}$. We consider the following biharmonic problem:

$$
\begin{gathered}
\Delta^{2} \Phi=0 \\
\left.\Phi\right|_{\partial \Omega}=-\left.\varphi\right|_{\partial \Omega},\left.\quad \frac{\partial \Phi}{\partial n}\right|_{\partial \Omega}=-\left.\frac{\partial \varphi}{\partial n}\right|_{\partial \Omega}, \\
\left.\Phi\right|_{\Gamma}=0,\left.\quad \Delta \Phi\right|_{\Gamma}=0 .
\end{gathered}
$$

Let $u^{\prime}=\nabla \Lambda(\chi \Phi)$; then $\Theta u=u+u^{\prime}$ is the desired operator. In fact, if $\left.u\right|_{\partial \Omega}=0$, then $\left.\varphi\right|_{\partial \Omega}=\left.\frac{\partial \varphi}{\partial n}\right|_{\partial \Omega}=0$, and $\Theta u=u$, so $\Theta$ is a projection. By the estimate of the elliptic problem and the trace theorem,

$$
\|\Phi\|_{s+2} \leq C\left(\|\varphi\|_{s+3 / 2, \partial \Omega}+\left\|\frac{\partial \varphi}{\partial n}\right\|_{s+1 / 2, \partial \Omega}\right) \leq C\|\varphi\|_{s+2} \leq C\|u\|_{s+1},
$$

hence

$$
\left\|u^{\prime}\right\|_{s+1} \leq C\|u\|_{s+1}
$$

which proves (1.5).

$\Phi$ can be obtained by the Galerkin scheme. To show that, we give a weak formulation of the above biharmonic problem. Let $-\Delta \Phi=\psi$; then $-\Delta \psi=0$. 
We take a test function $v \in H^{1}\left(\Omega^{\prime}\right),\left.v\right|_{\Gamma}=0$; then $\Phi \in H^{1}\left(\Omega^{\prime}\right),\left.\Phi\right|_{\partial \Omega}=$ $-\left.\varphi\right|_{\partial \Omega},\left.\Phi\right|_{\Gamma}=0$, and

$$
(\nabla \Phi, \nabla v)_{\Omega^{\prime}}-\left(\frac{\partial \Phi}{\partial n}, v\right)_{\partial \Omega}=(\psi, v)_{\Omega^{\prime}} .
$$

Let $\omega=-\nabla \Lambda u$; then $-\Delta \varphi=\omega$, and

$$
(\nabla \varphi, \nabla v)_{\Omega^{\prime}}-\left(\frac{\partial \varphi}{\partial n}, v\right)_{\partial \Omega}=(\omega, v)_{\Omega^{\prime}} .
$$

Adding up these relations and noting the boundary condition, we have

$$
(\nabla \Phi+\nabla \varphi, \nabla v)_{\Omega^{\prime}}=(\psi+\omega, v)_{\Omega^{\prime}} \quad \forall v \in H^{1}\left(\Omega^{\prime}\right),\left.v\right|_{\Gamma}=0
$$

We take another test function $v_{1} \in H_{0}^{1}\left(\Omega^{\prime}\right)$; then we have $\psi \in H^{1}\left(\Omega^{\prime}\right),\left.\psi\right|_{\Gamma}=$ 0 , and

$$
\left(\nabla \psi, \nabla v_{1}\right)=0 \quad \forall v_{1} \in H_{0}^{1}\left(\Omega^{\prime}\right) .
$$

(2.7), (2.8) is the desired weak formulation.

Finally, we list the definitions and some properties of the Helmholtz operator $P$ and Stokes operator $A$. It is known that $\left(L^{2}(\Omega)\right)^{2}=X \oplus G$, where $X=$ closure in $\left(L^{2}(\Omega)\right)^{2}$ of $\left\{u \in\left(C_{0}^{\infty}(\Omega)\right)^{2} ; \nabla \cdot u=0\right\}$ and $G=\left\{\nabla p ; p \in E^{1}(\Omega)\right\}$. $P$ is the orthogonal projection $P:\left(L^{2}(\Omega)\right)^{2} \rightarrow X$; consequently,

$$
\|P u\|_{0} \leq\|u\|_{0} \quad \forall u \in\left(L^{2}(\Omega)\right)^{2} .
$$

If $u \in\left(H^{s}(\Omega)\right)^{2}, s \geq 1$, then [9, Chapter 1, $\left.\S 2.4\right]$

$$
u=\nabla \varphi+v, \quad \nabla \cdot v=0,\left.\quad v \cdot n\right|_{\partial \Omega}=0,
$$

and $\varphi$ is the solution of

Like (2.6), we can obtain

$$
\begin{aligned}
-\Delta \varphi & =-\nabla \cdot u \\
\left.\frac{\partial \varphi}{\partial n}\right|_{\partial \Omega} & =\left.u \cdot n\right|_{\partial \Omega}
\end{aligned}
$$

$$
[\nabla \varphi]_{s} \leq C\|u\|_{s} \quad \forall s \geq 0,
$$

therefore $P:\left(H^{s}(\Omega)\right)^{2} \rightarrow\left(H^{s}(\Omega)\right)^{2}$.

We consider the Stokes equation

$$
\frac{\partial u}{\partial t}+\frac{1}{\rho} \nabla p=\nu \Delta u+f
$$

and conditions (1.2)-(1.4). Let $u=e^{\nu t} v, p=e^{\nu t} q$; then

$$
\frac{\partial v}{\partial t}+\frac{1}{\rho} \nabla q=\nu(\Delta v-v)+e^{-\nu t} f .
$$

The Stokes operator is defined as [7]: $A=-P \Delta+I$, with domain $D(A)=$ $X \cap\left\{u \in\left(H^{2}(\Omega)\right)^{2} ;\left.u\right|_{\partial \Omega}=0\right\}$. The solution $v$ can be expressed as

$$
v=e^{-\nu t A} u_{0}+\int_{0}^{t} e^{-\nu(t-\tau) A} P e^{-\nu \tau} f(\tau) d \tau,
$$


hence

$$
u=e^{-\nu t(A-I)} u_{0}+\int_{0}^{t} e^{-\nu(t-\tau)(A-I)} P f(\tau) d \tau
$$

We have [7]

$$
\begin{gathered}
\left\|A^{\alpha} e^{-t A}\right\| \leq C t^{-\alpha}, \quad \alpha \geq 0, \quad t>0, \\
C^{-1}\|u\|_{2 \alpha} \leq\left\|A^{\alpha} u\right\|_{0} \leq C\|u\|_{2 \alpha} \quad \forall u \in D\left(A^{\alpha}\right), \quad \alpha \geq 0 .
\end{gathered}
$$

And if $0 \leq s<\frac{1}{2}, u \in X \cap\left(H^{s}(\Omega)\right)^{2}$, then $u \in D\left(A^{s / 2}\right)$; if $1 \leq s<\frac{3}{2}$, $u \in D(A) \cap\left(H^{s+1}(\Omega)\right)^{2}$, then $u \in D\left(A^{(s+1) / 2}\right)[15]$.

\section{Solutions of the Stokes equation}

In this section we consider problems $(2.11),(1.2),(1.3),(1.4)$ and give some estimates. It is assumed that all functions appearing below belong to $L^{2}(\Omega)$.

Lemma 1. If $u$ is the solution of (2.11), (1.2), (1.3), (1.4), then

$$
\|u(t)\|_{0}^{2} \leq e^{t}\left(\left\|u_{0}\right\|_{0}^{2}+\int_{0}^{t}\|f(\tau)\|_{0}^{2} d \tau\right) .
$$

Proof. Taking the inner product of $(2.11)$ with $u$, we get $\left(u, \frac{\partial u}{\partial t}\right)=\nu(\Delta u, u)+$ $(f, u)$. Integrating by parts, we obtain $\frac{1}{2} \frac{d}{d t}\|u\|_{0}^{2}+\nu(\nabla u, \nabla u)=(f, u)$. Thus, $\frac{d}{d t}\|u\|_{0}^{2} \leq\|u\|_{0}^{2}+\|f\|_{0}^{2}$. By the Gronwall lemma, this gives (3.1).

Lemma 2. Let $\omega=-\nabla \Lambda u$, and let $u$ be the solution of (2.11), (1.2), (1.3), (1.4); then

$$
\frac{d}{d t}\|\omega(t)\|_{0}^{2} \leq \frac{1}{2 \nu}\|f\|_{0}^{2} .
$$

Proof. We apply the operator $-\nabla \Lambda$ to equation (2.11) and obtain

$$
\frac{\partial \omega}{\partial t}=\nu \Delta \omega-\nabla \Lambda f .
$$

The stream function $\psi$ is the solution of (2.1). Thus, the weak formulation is: find $\omega \in E^{1}(\Omega)$ and $\psi \in E_{0}^{1}(\Omega)$ such that

$$
\begin{gathered}
\frac{d}{d t}(\omega, v)+\nu(\nabla \omega, \nabla v)=-(\nabla \Lambda f, v)=(f, \nabla \Lambda v) \quad \forall v \in E_{0}^{1}(\Omega) \\
(\nabla \psi, \nabla \chi)=(\omega, \chi) \quad \forall \chi \in E^{1}(\Omega)
\end{gathered}
$$

We take $\chi \in E_{0}^{1}(\Omega)$; then by (3.4) and (3.5),

$$
\left(\nabla \frac{\partial \psi}{\partial t}, \nabla \chi\right)=\frac{d}{d t}(\nabla \psi, \nabla \chi)=\frac{d}{d t}(\omega, \chi)=(f, \nabla \Lambda \chi)-\nu(\nabla \omega, \nabla \chi) .
$$

Let $\chi=\frac{\partial \psi}{\partial t} ;$ then

$$
\left(\nabla \frac{\partial \psi}{\partial t}, \nabla \frac{\partial \psi}{\partial t}\right)=\left(f, \nabla \Lambda \frac{\partial \psi}{\partial t}\right)-\nu\left(\nabla \omega, \nabla \frac{\partial \psi}{\partial t}\right)
$$


(3.5) also yields

$$
\left(\nabla \frac{\partial \psi}{\partial t}, \nabla \chi\right)=\left(\frac{\partial \omega}{\partial t}, \chi\right) \quad \forall \chi \in E^{1}(\Omega) .
$$

Set $\chi=\omega$; then

$$
\left(\nabla \frac{\partial \psi}{\partial t}, \nabla \omega\right)=\left(\frac{\partial \omega}{\partial t}, \omega\right)
$$

Substitute this into (3.6) to obtain

$$
\left\|\nabla \frac{\partial \psi}{\partial t}\right\|_{0}^{2}+\nu\left(\frac{\partial \omega}{\partial t}, \omega\right)=\left(f, \nabla \Lambda \frac{\partial \psi}{\partial t}\right) \leq \frac{1}{4}\|f\|_{0}^{2}+\left\|\nabla \frac{\partial \psi}{\partial t}\right\|_{0}^{2} .
$$

Therefore,

which is $(3.2)$.

$$
\frac{\nu}{2} \frac{d}{d t}\|\omega\|_{0}^{2} \leq \frac{1}{4}\|f\|_{0}^{2}
$$

Lemma 3. If $u_{0} \in D(A) \cap\left(H^{s+1}(\Omega)\right)^{2}, 0 \leq s<\frac{3}{2}, f \in L^{\infty}\left(0, T ;\left(H^{1}(\Omega)\right)^{2}\right)$, and $u$ is the solution of (2.11), (1.2), (1.3), (1.4), then

$$
\|u(t)\|_{s+1} \leq C\left(\left\|u_{0}\right\|_{s+1}+\sup _{0 \leq \tau \leq T}\|f(\tau)\|_{1}\right) .
$$

Proof. We estimate the terms of (2.12). According to the statement at the end of $\S 2, u_{0} \in D\left(A^{(s+1) / 2}\right)$. By (2.13), (2.14),

$$
\begin{aligned}
\left\|e^{-\nu t(A-I)} u_{0}\right\|_{s+1} & \leq C\left\|A^{(s+1) / 2} e^{-\nu t A} e^{\nu t} u_{0}\right\|_{0}=C\left\|e^{-\nu t A} A^{(s+1) / 2} e^{\nu t} u_{0}\right\|_{0} \\
& \leq C\left\|A^{(s+1) / 2} e^{\nu t} u_{0}\right\|_{0} \leq C\left\|e^{\nu t} u_{0}\right\|_{s+1} \leq C\left\|u_{0}\right\|_{s+1} .
\end{aligned}
$$

Take a positive constant $r$ such that $s-1<r<\frac{1}{2}$; then $P f(\tau) \in D\left(A^{r / 2}\right)$ $\forall \tau \in[0, T]$, and

$$
\begin{aligned}
& \left\|\int_{0}^{t} e^{-\nu(t-\tau)(A-I)} P f(\tau) d \tau\right\|_{s+1} \leq C \int_{0}^{t}\left\|A^{(s+1) / 2} e^{-\nu(t-\tau)(A-I)} P f(\tau)\right\|_{0} d \tau \\
& \quad=C \int_{0}^{t}\left\|A^{(s+1-r) / 2} e^{-\nu(t-\tau)(A-I)} A^{r / 2} P f(\tau)\right\|_{0} d \tau \\
& \quad \leq C \int_{0}^{t}(\nu(t-\tau))^{-(s+1-r) / 2}\left\|e^{\nu(t-\tau)} A^{r / 2} P f(\tau)\right\|_{0} d \tau \\
& \quad \leq C \int_{0}^{t}(\nu(t-\tau))^{-(s+1-r) / 2}\|f(\tau)\|_{r} d \tau \\
& \quad \leq C \sup _{0 \leq \tau \leq T}\|f(\tau)\|_{1} .
\end{aligned}
$$

Now we apply scheme (1.6)-(1.13) to problem (2.11), (1.2), (1.3), (1.4). Equation (1.6) reduces to

$$
\frac{\partial \tilde{u}_{k}}{\partial t}+\frac{1}{\rho} \nabla \tilde{p}_{k}=f
$$


Applying the operator $P$ to $(3.8)$, we obtain $\partial \tilde{u}_{k} / \partial t=P f$, thus

$$
\tilde{u}_{k}(t)=u_{k}(i k-0)+\int_{i k}^{t} \operatorname{Pf}(\tau) d \tau, \quad i k \leq t<(i+1) k .
$$

By induction and (2.12), it can be proved that

$$
\begin{aligned}
u_{k}(t)= & e^{-\nu t(A-I)} u_{0}+\sum_{i=0}^{[t / k]} e^{-\nu(t-i k)(A-I)} \int_{i k}^{(i+1) k} \Theta P f(\tau) d \tau \\
& +\sum_{i=0}^{[t / k]-1} \int_{i k}^{(i+1) k} e^{-\nu(t-\tau)(A-I)} \frac{1}{k} \int_{i k}^{(i+1) k}(I-\Theta) P f(\zeta) d \zeta d \tau \\
& +\int_{[t / k] k}^{t} e^{-\nu(t-\tau)(A-I)} \frac{1}{k} \int_{[t / k] k}^{([t / k]+1) k}(I-\Theta) P f(\zeta) d \zeta d \tau,
\end{aligned}
$$

where [ ] denotes the integral part of a number.

Lemma 4. If $u_{0} \in D(A) \cap\left(H^{s+1}(\Omega)\right)^{2}, 0 \leq s<\frac{3}{2}, f \in L^{\infty}\left(0, T ;\left(H^{1}(\Omega)\right)^{2}\right)$, then

$$
\left\|u_{k}(j k-0)\right\|_{s+1} \leq C\left(\left\|u_{0}\right\|_{s+1}^{\cdot}+\sup _{0 \leq \tau<j k}\|f(\tau)\|_{1}\right) .
$$

Proof. We estimate the second term of (3.10); the estimate of other terms is similar. Let $r$ be a positive constant such that $s-1<r<\frac{1}{2}$; then

$$
\begin{aligned}
& \left\|\sum_{i=0}^{j-1} e^{-\nu(j-i) k(A-I)} \int_{i k}^{(i+1) k} \Theta P f(\tau) d \tau\right\|_{s+1} \\
& \quad \leq C\left\|\sum_{i=0}^{j-1} A^{(s+1) / 2} e^{-\nu(j-i) k(A-I)} \int_{i k}^{(i+1) k} \Theta P f(\tau) d \tau\right\|_{0} \\
& \quad=C\left\|\sum_{i=0}^{j-1} A^{(s+1-r) / 2} e^{-\nu(j-i) k(A-I)} A^{r / 2} \int_{i k}^{(i+1) k} \Theta P f(\tau) d \tau\right\|_{0} \\
& \leq C \sum_{i=0}^{j-1}(\nu(j-i) k)^{-(s+1-r) / 2} \int_{i k}^{(i+1) k}\left\|e^{\nu(j-i) k} A^{r / 2} \Theta P f(\tau)\right\|_{0} d \tau \\
& \quad \leq C \sup _{0 \leq \tau<j k}\|f(\tau)\|_{1} \sum_{i=0}^{j-1}(\nu(j-i) k)^{-(s+1-r) / 2} k \\
& \quad \leq C \sup _{0 \leq \tau<j k}\|f(\tau)\|_{1} \int_{0}^{j k}(\nu(j k-\tau))^{-(s+1-r) / 2} d \tau \\
& \leq C \sup _{0 \leq \tau<j k}\|f(\tau)\|_{1} \cdot \square
\end{aligned}
$$

Lemma 5. If $u_{0} \in D(A) \cap\left(H^{s+1}(\Omega)\right)^{2}, 0 \leq s<\frac{3}{2}, f \in L^{\infty}\left(0, T ;\left(H^{3}(\Omega)\right)^{2}\right) \cap$ $W^{1, \infty}\left(0, T ;\left(H^{1}(\Omega)\right)^{2}\right), u$ is the solution of problem $(2.11),(1.2),(1.3),(1.4)$, 
$\frac{\partial u}{\partial t} \in L^{\infty}\left(0, T ;\left(H^{s+1}(\Omega)\right)^{2}\right)$, and $u_{k}, \tilde{u}_{k}$ are the solutions of problems (3.8), (1.7)-(1.13), then

$$
\sup _{0 \leq t \leq T}\left(\left\|u(t)-u_{k}(t)\right\|_{s+1},\left\|u(t)-\tilde{u}_{k}(t)\right\|_{s+1}\right) \leq C_{0} k
$$

Proof. By (2.12), (3.10) we have

$$
\begin{aligned}
u(t)-u_{k}(t)= & \sum_{i=0}^{[t / k]-1} \int_{i k}^{(i+1) k}\left(e^{-\nu(t-\tau)(A-I)}-e^{-\nu(t-i k)(A-I)}\right) \Theta P f(\tau) d \tau \\
& +\int_{[t / k] k}^{t}\left(e^{-\nu(t-\tau)(A-I)}-e^{-\nu(t-[t / k] k)(A-I)}\right) \Theta P f(\tau) d \tau \\
& -\int_{t}^{([t / k]+1) k} e^{-\nu(t-[t / k] k)(A-I)} \Theta P f(\tau) d \tau \\
& +\sum_{i=0}^{[t / k]-1} \int_{i k}^{(i+1) k} e^{-\nu(t-\tau)(A-I)} \frac{1}{k} \\
& +\int_{[t / k] k}^{t} e^{-\nu(t-\tau)(A-I)} \frac{1}{k} \int_{[t / k] k}^{([t / k]+1) k}(I-\Theta) P(f(\tau)-f(\zeta)) d \zeta d \tau .
\end{aligned}
$$

We estimate the terms in (3.12). With regard to the first term,

$$
\begin{aligned}
& I_{1}=\left\|\sum_{i} \int_{i k}^{(i+1) k}\left(e^{-\nu(t-\tau)(A-I)}-e^{-\nu(t-i k)(A-I)}\right) \Theta P f(\tau) d \tau\right\|_{S+1} \\
& \leq C\left\|\sum_{i} \int_{i k}^{(i+1) k} A^{(s+1) / 2} e^{-\nu(t-\tau)(A-I)}\left(I-e^{-\nu(\tau-i k)(A-I)}\right) \Theta P f(\tau) d \tau\right\|_{0} \\
&=C \| \sum_{i} \int_{i k}^{(i+1) k} A^{(s+1) / 2} \nu(A-I) e^{-\nu(t-\tau)(A-I)} \\
& \cdot \int_{0}^{\tau-i k} e^{-\nu \zeta(A-I)} d \zeta \cdot \Theta P f(\tau) d \tau \|_{0} .
\end{aligned}
$$

Taking a constant $s_{1}, s<s_{1}<\frac{3}{2}$, we get

$$
\begin{aligned}
I_{1} & \leq C \| \sum_{i} \int_{i k}^{(i+1) k} A^{\left(s-s_{1}\right) / 2}(A-I) e^{-\nu(t-\tau) A} A^{\left(s_{1}+1\right) / 2} e^{\nu(t-\tau)} \\
& \cdot \int_{0}^{\tau-i k} e^{-\nu \zeta(A-I)} d \zeta \cdot \Theta P f(\tau) d \tau \|_{0} \\
& \leq C \sum_{i} \int_{i k}^{(i+1) k}(t-\tau)^{-1+\left(s_{1}-s\right) / 2} \int_{0}^{\tau-i k}\left\|A^{\left(s_{1}+1\right) / 2} \Theta P f(\tau)\right\|_{0} d \zeta d \tau \\
& \leq C \sup _{0 \leq \tau \leq T}\|\Theta P f(\tau)\|_{s_{1}+1} k \int_{0}^{t}(t-\tau)^{-1+\left(s_{1}-s\right) / 2} d \tau \\
& \leq C k \sup _{0 \leq \tau \leq T}\|f(\tau)\|_{s_{1}+1} .
\end{aligned}
$$


With regard to the fourth term, we take a positive constant $r, s-1<r<\frac{1}{2}$; then applying (2.13), (2.14), we get

$$
\begin{aligned}
I_{4} & =\left\|\sum_{i} \int_{i k}^{(i+1) k} e^{-\nu(t-\tau)(A-I)} \frac{1}{k} \int_{i k}^{(i+1) k}(I-\Theta) P(f(\tau)-f(\zeta)) d \zeta d \tau\right\|_{s+1} \\
& =\left\|\sum_{i} \int_{i k}^{(i+1) k} e^{-\nu(t-\tau)(A-I)} \frac{1}{k} \int_{i k}^{(i+1) k} \int_{\zeta}^{\tau}(I-\Theta) P f^{\prime}(\xi) d \xi d \zeta d \tau\right\|_{s+1} \\
& \leq C\left\|\sum_{i} \int_{i k}^{(i+1) k} A^{(s+1) / 2} e^{-\nu(t-\tau)(A-I)} \frac{1}{k} \int_{i k}^{(i+1) k} \int_{\zeta}^{\tau}(I-\Theta) P f^{\prime}(\xi) d \xi d \zeta d \tau\right\|_{0} \\
& \leq C \sum_{i} \int_{i k}^{(i+1) k}(\nu(t-\tau))^{-(s+1-r) / 2} \frac{1}{k} \\
& \leq C \int_{0}^{t}(t-\tau)^{-(s+1-r) / 2} d \tau \cdot \int_{i}^{(i+1) k} \int_{i k}^{(i+1) k}\left\|(I-\Theta) P f^{\prime}(\xi)\right\|_{r} d \xi d \zeta d \tau \\
& \leq C k \sup _{0 \leq \xi \leq T}\left\|f^{\prime}(\xi)\right\|_{1} \cdot
\end{aligned}
$$

The rest of the terms can be estimated in a similar way, and therefore we get the desired estimate of $\left\|u(t)-u_{k}(t)\right\|_{s+1}$.

Now we estimate $\left\|u(t)-\tilde{u}_{k}(t)\right\|_{s+1}$. Since $\left\|\frac{\partial u}{\partial t}\right\|_{s+1}$ is bounded, we have

$$
\|u(t)-u(i k)\|_{s+1} \leq C_{0} k, \quad t \in[i k,(i+1) k) \text {. }
$$

By (3.9),

Therefore,

$$
\left\|\tilde{u}_{k}(t)-u_{k}(i k-0)\right\|_{s+1} \leq C k \sup _{i k \leq \tau \leq t}\|f(\tau)\|_{s+1}
$$

$$
\left\|u(t)-\tilde{u}_{k}(t)\right\|_{s+1} \leq C_{0} k+\left\|u(i k)-u_{k}(i k-0)\right\|_{s+1} \leq C_{0} k .
$$

\section{Solutions of the Euler equation}

We consider

$$
\begin{gathered}
\frac{\partial u}{\partial t}+(u \cdot \nabla) u+\frac{1}{\rho} \nabla p=f, \\
\nabla \cdot u=0, \\
\left.u \cdot n\right|_{x \in \partial \Omega}=0, \\
\left.u\right|_{t=0}=u_{0}(x) .
\end{gathered}
$$

The existence and uniqueness theorem has been proved by several authors. We apply the result of [12] here. Although only bounded domains were considered in [12], a slight modification of the proof will yield the result for unbounded domains. In brief, if $u_{0} \in\left(H^{m}(\Omega)\right)^{2}, f \in L^{1}\left(0, T ;\left(H^{m}(\Omega)\right)^{2}\right), m \geq 3$, then the local solution $u \in L^{\infty}\left(0, T ;\left(H^{m}(\Omega)\right)^{2}\right)$. 
Lemma 6. If the integer $m \geq \max (3, s+1), s \geq-1,\left\|u_{0}\right\|_{m} \leq M_{1}, u_{0} \in X$, then there exists a constant $C>0$ such that if

$$
k_{0}=\frac{1}{C\left(M_{1}+\sup _{0 \leq t \leq T}\|f(t)\|_{m}+1\right)}
$$

and $0 \leq t \leq k_{0}$, then the solution $u$ of (4.1)-(4.4) satisfies

$$
\|u\|_{s+1} \leq C_{1}\left(\left\|u_{0}\right\|_{s+1}+1\right),
$$

where the constant $C_{1}$ depends only on the constant $T$ and $\sup _{0 \leq t \leq T}\|f(t)\|_{m}$. Proof. From (4.1), (4.2) we get [12]

$$
\begin{gathered}
\frac{1}{\rho} \Delta p=\nabla \cdot f-\nabla \cdot((u \cdot \nabla) u), \\
\left.\frac{\partial p}{\partial n}\right|_{x \in \partial \Omega}=\rho f \cdot n+\sum_{i, j} \phi_{i j} u_{i} u_{j},
\end{gathered}
$$

where $\phi_{i j}$ are bounded functions. Analogously to the proof in [12], it can be proved that $p \in E^{m+1}(\Omega)$, and

$$
\|\nabla p\|_{m} \leq C\left\{\|f(t)\|_{m}+\|u(t)\|_{m}^{2}\right\} .
$$

By (4.1), (4.7) we get

$$
\frac{d}{d t}\|u\|_{m} \leq C\left(\|u\|_{m}^{2}+\|f\|_{m}\right)
$$

Therefore, $\|u(t)\|_{m} \leq y(t)$, where $y(t)$ is the solution of the initial value problem $y^{\prime}=C y^{2}+C\|f(t)\|_{m}, y(0)=\left\|u_{0}\right\|_{m}$. We take

$$
M=3\left(\left\|u_{0}\right\|_{m}+\sup _{0 \leq t \leq T}\|f(t)\|_{m}\right)
$$

and impose the restriction $|y| \leq M$. Then

$$
0 \leq y(t) \leq\left\|u_{0}\right\|_{m}+C \int_{0}^{t}\|f(\tau)\|_{m} d \tau+C M \int_{0}^{t} y(\tau) d \tau .
$$

By the Gronwall lemma,

$$
y(t) \leq e^{C M t}\left(\left\|u_{0}\right\|_{m}+C \int_{0}^{t}\|f(\tau)\|_{m} d \tau\right) .
$$

We take $t>0$ such that $t \leq 1 /(C M+C)$; then $C M t \leq 1$ and $C t \leq 1$. (4.8) yields $y(t) \leq M$. Comparing the upper bound of $t$ with (4.5), it will suffice that $t \leq k_{0}$ for a suitable constant $C$.

We consider the auxiliary linear problem

$$
\begin{gathered}
\frac{\partial \tilde{u}}{\partial t}+(u \cdot \nabla) \tilde{u}+\nabla \tilde{\pi}=\tilde{f}, \quad \nabla \cdot \tilde{u}=0, \\
\left.\tilde{u} \cdot n\right|_{x \in \partial \Omega}=0,\left.\quad \tilde{u}\right|_{t=0}=\tilde{u}_{0}(x) .
\end{gathered}
$$


When $\tilde{u}_{0}=u_{0}, \tilde{f}=f$, then by uniqueness $\tilde{u}=u$. In a manner similar to [12], we get

$$
\|\nabla \tilde{\pi}(t)\|_{m} \leq C\left(\|\tilde{f}(t)\|_{m}+\|u(t)\|_{m}\|\tilde{u}(t)\|_{m}\right)
$$

then we can prove

$$
\frac{1}{2} \frac{d}{d t}\|\tilde{u}(t)\|_{m}^{2} \leq C\left(\|\tilde{f}(t)\|_{m}+\|u(t)\|_{m}\|\tilde{u}(t)\|_{m}\right)\|\tilde{u}(t)\|_{m}
$$

But $\|u(t)\|_{m} \leq M$, and by Gronwall's lemma

$$
\|\tilde{u}(t)\|_{m} \leq e^{C M t}\left(\left\|\tilde{u}_{0}\right\|_{m}+C k_{0} \sup _{0 \leq \tau \leq t}\|\tilde{f}(\tau)\|_{m}\right) .
$$

By (4.5),

$$
\|\tilde{u}(t)\|_{m} \leq e\left(\left\|\tilde{u}_{0}\right\|_{m}+\sup _{0 \leq \tau \leq t}\|\tilde{f}(\tau)\|_{m}\right)
$$

under the restriction $t \leq k_{0}$. Taking the inner product of $\tilde{u}$ with equation (4.9), we get $\left(\frac{\partial \tilde{u}}{\partial t}, \tilde{u}\right)=(\tilde{f}, \tilde{u})$, hence

$$
\|\tilde{u}(t)\|_{0} \leq\left\|\tilde{u}_{0}\right\|_{0}+\int_{0}^{t}\|\tilde{f}(\tau)\|_{0} d \tau
$$

The mapping $\left(\tilde{u}_{0}, \tilde{f}\right) \rightarrow \tilde{u}$ is linear, and by the interpolation theorem and (4.10),

$$
\|\tilde{u}(t)\|_{s+1} \leq C\left(\left\|\tilde{u}_{0}\right\|_{s+1}+\sup _{0 \leq \tau \leq t}\|\tilde{f}(\tau)\|_{s+1}\right) .
$$

Letting $\tilde{u}_{0}=u_{0}, \tilde{f}=f$, one obtains (4.6).

Now, $u$ is assumed to be an arbitrary vector function which belongs to $L^{\infty}\left(0, T ;\left(W^{2, \infty}(\Omega)\right)^{2}\right)$, and with $u(\cdot, t) \in X, u_{0} \in\left(H^{1}(\Omega)\right)^{2} \cap X$ we let $\omega$ be the solution of

$$
\frac{\partial \omega}{\partial t}+u \cdot \nabla \omega=-\nabla \Lambda f \equiv F,\left.\quad \omega\right|_{t=0}=-\nabla \Lambda u_{0} \equiv \omega_{0} .
$$

We denote by $\xi(y, t ; \tau)$ the characteristic which satisfies

$$
\frac{\partial}{\partial t} \xi(y, t ; \tau)=u(\xi(y, t ; \tau), t), \quad \xi(y, \tau ; \tau)=y .
$$

Let $\psi \in E_{0}^{2}(\Omega)$ be the stream function corresponding to $u_{0}$, and

$$
\Psi(y)=\psi(\xi(y, 0 ; t)), \quad \theta=-\Delta \Psi .
$$

Then we have the following lemma.

Lemma 7. If $u_{0} \in D(A)$, then

$$
\|\theta(t)-\omega(t)\|_{0} \leq C_{2} t\left\|u_{0}\right\|_{1}+\int_{0}^{t}\|F(\tau)\|_{0} d \tau,
$$


where the constant $C_{2}$ depends only on the domain $\Omega$ and the function $u$. Proof. We have

$$
\begin{aligned}
\Delta \Psi= & \partial_{1}^{2} \psi \cdot\left|\nabla \xi_{1}\right|^{2}+2 \partial_{1} \partial_{2} \psi \cdot\left(\partial_{1} \xi_{1} \cdot \partial_{1} \xi_{2}+\partial_{2} \xi_{1} \cdot \partial_{2} \xi_{2}\right)+\partial_{2}^{2} \psi \cdot\left|\nabla \xi_{2}\right|^{2} \\
& +\partial_{1} \psi \cdot \Delta \xi_{1}+\partial_{2} \psi \cdot \Delta \xi_{2}, \\
\partial_{i} \xi_{j}= & \delta_{i j}+O(t), \quad \Delta \xi=O(t),
\end{aligned}
$$

hence

$$
-\Delta \Psi=\omega_{0}(\xi(y, 0 ; t))+R_{1},
$$

where

$$
\left\|R_{1}\right\|_{0}=O(t)[\psi]_{2} .
$$

Integrating equation (4.11) along characteristics, we obtain

$$
\omega(x, t)=\omega_{0}(\xi(x, 0 ; t))+\int_{0}^{t} F(\xi(x, \zeta ; t), \zeta) d \zeta .
$$

Since the mapping $x \rightarrow \xi(x, \tau ; t)$ is measure-preserving, we get

$$
\begin{aligned}
\left\|\omega(t)-\omega_{0}(\xi(\cdot, 0 ; t))\right\|_{0} & =\left\|\int_{0}^{t} F(\xi(\cdot, \zeta ; t), \zeta) d \zeta\right\|_{0} \\
& \leq \int_{0}^{t}\|F(\xi(\cdot, \zeta ; t), \zeta)\|_{0} d \zeta=\int_{0}^{t}\|F(\zeta)\|_{0} d \zeta .
\end{aligned}
$$

By (4.13), $\theta(t)=\omega(t)+R_{1}+R_{2}$, and

$$
\left\|R_{2}\right\|_{0} \leq \int_{0}^{t}\|F(\zeta)\|_{0} d \zeta .
$$

Then (4.14), (4.15) give (4.12).

\section{SOME ESTIMATES FOR THE VISCOUS SPLITTING SCHEME}

In this section we give some estimates for the solutions of the scheme (1.6)(1.13). We always denote by $u, \omega$ the solution of problem (1.1)-(1.4), and by $\omega_{k}, \tilde{\omega}_{k}$ the vorticity corresponding to $u_{k}, \tilde{u}_{k}$. We recall that we assume $u_{0} \in D(A) \cap\left(H^{3}(\Omega)\right)^{2}, f \in L^{\infty}\left(0, T ;\left(H^{3}(\Omega)\right)^{2}\right) \cap W^{1, \infty}\left(0, T ;\left(H^{1}(\Omega)\right)^{2}\right)$ and $u \in L^{\infty}\left(0, T ;\left(H^{4}(\Omega)\right)^{2}\right) \cap W^{1, \infty}\left(0, T ;\left(H^{5 / 2}(\Omega)\right)^{2}\right)$.

Lemma 8. If $1<s<\frac{3}{2}$, and if there is a constant $M_{0}$ such that

$$
\left\|\tilde{u}_{k}(t)\right\|_{1} \leq M_{0}, \quad 0 \leq t \leq T,
$$

and constants $C_{1}, k_{0}>0$ such that

$$
\left\|\tilde{u}_{k}(t)\right\|_{s+1} \leq C_{1}\left(\left\|\tilde{u}_{k}(i k)\right\|_{s+1}+1\right), \quad i k \leq t<(i+1) k,
$$

for $0<k \leq k_{0}$, then

$$
\sup _{0 \leq t \leq T}\left\|\tilde{u}_{k}(t)\right\|_{s+1} \leq M_{2}
$$


for $0<k \leq k_{0}$, where the constant $M_{2}$ depends only on the domain $\Omega$, the operator $\Theta$, the constants $C_{1}, M_{0}, T, s, \nu$, and the functions $f, u_{0}$.

Proof. We denote by $C_{3}$ a generic constant depending only on the domain $\Omega$, the operator $\Theta$, the constants $C_{1}, T, s, \nu$, and the functions $f, u_{0}$. Set $f_{1}(\tau)=f(\tau)-\left(\tilde{u}_{k} \cdot \nabla\right) \tilde{u}_{k} ;$ then by Lemma 4 ,

$$
\left\|u_{k}(j k-0)\right\|_{s+1} \leq C\left(\left\|u_{0}\right\|_{s+1}+\sup _{0 \leq \tau<j k}\left\|f_{1}(\tau)\right\|_{1}\right) .
$$

The norm of the nonlinear term has an upper bound

$$
\left\|\left(\tilde{u}_{k} \cdot \nabla\right) \tilde{u}_{k}\right\|_{1} \leq C\left(\left\|\tilde{u}_{k}\right\|_{1,4}^{2}+\left\|\tilde{u}_{k}\right\|_{0, \infty}\left\|\tilde{u}_{k}\right\|_{2}\right) \text {. }
$$

We take a constant $q, 1<q<s$. Then using the imbedding theorem [1, Theorem 7.57],

$$
\left\|f_{1}(\tau)\right\|_{1} \leq\|f(\tau)\|_{1}+C\left(\left\|\tilde{u}_{k}\right\|_{3 / 2}^{2}+\left\|\tilde{u}_{k}\right\|_{q}\left\|\tilde{u}_{k}\right\|_{2}\right),
$$

and by the interpolation inequality [10, Chapter 1, Remark 9.1],

$$
\begin{aligned}
\left\|f_{1}(\tau)\right\|_{1} \leq & \|f(\tau)\|_{1}+C\left(\left\|\tilde{u}_{k}\right\|_{1}^{2-1 / s} \cdot\left\|\tilde{u}_{k}\right\|_{s+1}^{1 / s}\right. \\
& \left.+\left\|\tilde{u}_{k}\right\|_{1}^{1-(q-1) / s}\left\|\tilde{u}_{k}\right\|_{s+1}^{(q-1) / 2}\left\|\tilde{u}_{k}\right\|_{1}^{1-1 / s}\left\|\tilde{u}_{k}\right\|^{1 / s}\right) \\
= & \|f(\tau)\|_{1}+C\left(\left\|\tilde{u}_{k}\right\|_{1}^{2-1 / s}\left\|\tilde{u}_{k}\right\|_{s+1}^{1 / s}+\left\|\tilde{u}_{k}\right\|_{1}^{2-q / s}\left\|\tilde{u}_{k}\right\|_{s+1}^{q / s} .\right.
\end{aligned}
$$

Hence,

$$
\left\|u_{k}(j k-0)\right\|_{s+1} \leq C_{3}+C \sup _{0 \leq \tau<j k}\left(\left\|\tilde{u}_{k}\right\|_{1}^{2-1 / s}\left\|\tilde{u}_{k}\right\|_{s+1}^{1 / s}+\left\|\tilde{u}_{k}\right\|_{1}^{2-q / s}\left\|\tilde{u}_{k}\right\|_{s+1}^{q / s}\right) .
$$

By (5.2) and initial condition (1.9) we obtain

$$
\left\|\tilde{u}_{k}(t)\right\|_{s+1} \leq C_{3}+C_{3} \sup _{0 \leq \tau \leq T}\left(\left\|\tilde{u}_{k}\right\|_{1}^{2-1 / s}\left\|\tilde{u}_{k}\right\|_{s+1}^{1 / s}+\left\|\tilde{u}_{k}\right\|_{1}^{2-q / s}\left\|\tilde{u}_{k}\right\|_{s+1}^{q / s}\right)+C_{1} .
$$

Taking the supremum of the left-hand side and applying (5.1), we get

$$
\sup _{0 \leq t \leq T}\left\|\tilde{u}_{k}\right\|_{s+1} \leq C_{3}+C_{3}\left(M_{0}^{2-1 / s} \sup _{0 \leq t \leq T}\left\|\tilde{u}_{k}\right\|_{s+1}^{1 / s}+M_{0}^{2-q / s} \sup _{0 \leq t \leq T}\left\|\tilde{u}_{k}\right\|_{s+1}^{q / s}\right)+C_{1} .
$$

Then (5.3) follows.

If we replace $\left(\tilde{u}_{k} \cdot \nabla\right) \tilde{u}_{k}$ in equation $(1.6)$ by $(u \cdot \nabla) u$, then it becomes a linear equation

$$
\frac{\partial \tilde{u}_{k}}{\partial t}+\frac{1}{\rho} \nabla \tilde{p}_{k}=f-(u \cdot \nabla) u
$$

The solutions of problem (5.5), (1.7)-(1.13) are denoted by $\tilde{u}^{*}, \tilde{p}^{*}, u^{*}, p^{*}$. Let $\tilde{\omega}^{*}, \omega^{*}$ be the associated vorticities. By Lemma 5 , for any $0 \leq s^{\prime}<\frac{3}{2}$,

$$
\sup _{0 \leq t \leq T}\left(\left\|u(t)-u^{*}(t)\right\|_{s^{\prime}+1},\left\|u(t)-\tilde{u}^{*}(t)\right\|_{s^{\prime}+1}\right) \leq C_{0} k .
$$


Lemma 9. If $1<s<\frac{3}{2},\left\|\tilde{u}_{k}\right\|_{s+1} \leq M_{3}$, then

(5.7) $\left\|(I-\Theta)\left(\tilde{u}^{*}-\tilde{u}_{k}\right)((i+1) k-0)\right\|_{1} \leq C_{4} k\left(\sup _{i k \leq \tau<(i+1) k}\left\|\left(\tilde{u}^{*}-\tilde{u}_{k}\right)(\tau)\right\|_{1}+k\right)$,

where the constant $C_{4}$ depends only on the domain $\Omega$, the operator $\Theta$, the constants $s, \nu, T, M_{3}$, the functions $f, u_{0}$, and the solution $u$ of (1.1)(1.4).

Proof. We denote by $C_{4}$ a generic constant which possesses the above property. By (5.5) and (1.6),

$$
\frac{\partial \tilde{\omega}^{*}}{\partial t}+u \cdot \nabla \omega=F, \quad \frac{\partial \tilde{\omega}_{k}}{\partial t}+\tilde{u}_{k} \cdot \nabla \tilde{\omega}_{k}=F .
$$

On subtracting the two equations, we obtain

$$
\frac{\partial\left(\tilde{\omega}^{*}-\tilde{\omega}_{k}\right)}{\partial t}+u \cdot \nabla\left(\tilde{\omega}^{*}-\tilde{\omega}_{k}\right)=u \cdot \nabla\left(\tilde{\omega}^{*}-\omega\right)-\left(u-\tilde{u}_{k}\right) \cdot \nabla \tilde{\omega}_{k} .
$$

By Lemma 7 ,

$$
\begin{aligned}
\| \theta- & \left(\tilde{\omega}^{*}-\tilde{\omega}_{k}\right)((i+1) k-0) \|_{0} \\
\leq & C_{4} k\left\|\left(\tilde{u}^{*}-\tilde{u}_{k}\right)(i k)\right\|_{1} \\
& \quad+\int_{i k}^{(i+1) k}\left\|u \cdot \nabla\left(\tilde{\omega}^{*}-\omega\right)-\left(u-\tilde{u}_{k}\right) \cdot \nabla \tilde{\omega}_{k}\right\|_{0} d \tau,
\end{aligned}
$$

where $\theta=-\Delta \Psi, \Psi(y)=\psi(\xi(y, i k ;(i+1) k))$, and $\psi$ is the stream function corresponding to $\left(\tilde{u}^{*}-\tilde{u}_{k}\right)(i k)$.

We estimate the integrand. By (5.6),

$$
\left\|u \cdot \nabla\left(\tilde{\omega}^{*}-\omega\right)\right\|_{0} \leq C_{4}\left\|\tilde{u}^{*}-u\right\|_{2} \leq C_{4} k .
$$

Let $p=2 /(2-s)$ and $q=2 /(s-1)$; then

$$
\begin{aligned}
\left\|\left(u-\tilde{u}_{k}\right) \cdot \nabla \tilde{\omega}_{k}\right\|_{0} & =\left(\int_{\Omega}\left|\left(u-\tilde{u}_{k}\right) \cdot \nabla \tilde{\omega}_{k}\right|^{2} d x\right)^{1 / 2} \\
& \leq\left(\int_{\Omega}\left|\nabla \tilde{\omega}_{k}\right|^{p} d x\right)^{1 / p}\left(\int_{\Omega}\left|u-\tilde{u}_{k}\right|^{q} d x\right)^{1 / q} \\
& \leq\left\|\tilde{\omega}_{k}\right\|_{1, p}\left\|u-\tilde{u}_{k}\right\|_{0, q} .
\end{aligned}
$$

Using the imbedding theorem and (5.6),

$$
\begin{gathered}
\left\|\tilde{\omega}_{k}\right\|_{1, p} \leq C\left\|\tilde{\omega}_{k}\right\|_{s}, \\
\left\|u-\tilde{u}_{k}\right\|_{0, q} \leq C\left\|u-\tilde{u}_{k}\right\|_{1} \leq C_{0}\left(\left\|\tilde{u}^{*}-\tilde{u}_{k}\right\|_{1}+k\right) .
\end{gathered}
$$

Therefore,

$$
\left\|u \cdot \nabla\left(\tilde{\omega}^{*}-\omega\right)-\left(u-\tilde{u}_{k}\right) \cdot \nabla \tilde{\omega}_{k}\right\|_{0} \leq C_{4}\left(\left\|\tilde{u}^{*}-\tilde{u}_{k}\right\|_{1}+k\right) .
$$

Substituting (5.10) into (5.9), we obtain

$$
\left\|\theta-\left(\tilde{\omega}^{*}-\tilde{\omega}_{k}\right)((i+1) k-0)\right\|_{0} \leq C_{4} k\left(\sup _{i k \leq \tau<(i+1) k}\left\|\tilde{u}^{*}(\tau)-\tilde{u}_{k}(\tau)\right\|_{1}+k\right) .
$$


On subtracting equations (5.5) and (1.6), we have

$$
\frac{\partial\left(\tilde{u}^{*}-\tilde{u}_{k}\right)}{\partial t}+\frac{1}{\rho} \nabla\left(\tilde{p}^{*}-\tilde{p}_{k}\right)=\left(\tilde{u}_{k} \cdot \nabla\right) \tilde{u}_{k}-(u \cdot \nabla) u \equiv \mathscr{F},
$$

hence

$$
\begin{gathered}
\left(\tilde{u}^{*}-\tilde{u}_{k}\right)(t)-\left(\tilde{u}^{*}-\tilde{u}_{k}\right)(i k) \\
=-\frac{1}{\rho} \int_{i k}^{t} \nabla\left(\tilde{p}^{*}-\tilde{p}_{k}\right) d \tau+\int_{i k}^{t} \mathscr{F} d \tau \\
\left\|\left(\tilde{u}^{*}-\tilde{u}_{k}\right)(t)-\left(\tilde{u}^{*}-\tilde{u}_{k}\right)(i k)\right\|_{0} \\
\leq \frac{1}{\rho} \int_{i k}^{t}\left\|\nabla\left(\tilde{p}^{*}-\tilde{p}_{k}\right)\right\|_{0} d \tau+\int_{i k}^{t}\|\mathscr{F}\|_{0} d \tau .
\end{gathered}
$$

Similarly as in the proof of Lemma 6 , it can be shown that $\tilde{p}^{*}-\tilde{p}_{k}$ is the solution of

$$
\begin{aligned}
\frac{1}{\rho} \Delta\left(\tilde{p}^{*}-\tilde{p}_{k}\right) & =\nabla \cdot \mathscr{F} \\
\left.\frac{\partial\left(\tilde{p}^{*}-\tilde{p}_{k}\right)}{\partial n}\right|_{x \in \partial \Omega} & =\sum_{i, j} \phi_{i j}\left(\left(\tilde{u}_{k}\right)_{i}\left(\tilde{u}_{k}\right)_{j}-u_{i} u_{j}\right) \\
& =\sum_{i, j} \phi_{i j}\left(\left(\tilde{u}_{k}\right)_{i}\left(\tilde{u}_{k}-u\right)_{j}-\left(u-\tilde{u}_{k}\right)_{i} u_{j}\right) .
\end{aligned}
$$

In weak formulation, $\tilde{p}^{*}-\tilde{p}_{k} \in E^{1}(\Omega)$, and

$$
\begin{aligned}
& \left(\nabla\left(\tilde{p}^{*}-\tilde{p}_{k}\right), \nabla v\right)_{\Omega}+\left(\sum_{i, j} \phi_{i j}\left(\left(\tilde{u}_{k}\right)_{i}\left(\tilde{u}_{k}-u\right)_{j}-\left(u-\tilde{u}_{k}\right)_{i} u_{j}\right), v\right)_{\partial \Omega} \\
& =(\rho \mathscr{F}, \nabla v)_{\Omega} \quad \forall v \in E^{1}(\Omega) .
\end{aligned}
$$

We may assume that $\left(\tilde{p}^{*}-\tilde{p}_{k}, 1\right)_{\partial \Omega}=0$; then

$$
\left\|\tilde{p}^{*}-\tilde{p}_{k}\right\|_{0, \partial \Omega} \leq C\left[\tilde{p}^{*}-\tilde{p}_{k}\right]_{1} .
$$

Taking $v=\tilde{p}^{*}-\tilde{p}_{k}$ we get

$$
\begin{aligned}
{\left[\tilde{p}^{*}-\tilde{p}_{k}\right]_{1}^{2} \leq } & \left\|\sum_{i, j} \phi_{i j}\left(\left(\tilde{u}_{k}\right)_{i}\left(\tilde{u}_{k}-u\right)_{j}-\left(u-\tilde{u}_{k}\right)_{i} u_{j}\right)\right\|_{0, \partial \Omega}\left\|\tilde{p}^{*}-\tilde{p}_{k}\right\|_{0, \partial \Omega} \\
& +\|\rho \mathscr{F}\|_{0}\left[\tilde{p}^{*}-\tilde{p}_{k}\right]_{1},
\end{aligned}
$$

thus

$$
\left[\tilde{p}^{*}-\tilde{p}_{k}\right]_{1} \leq C\left\|\sum_{i, j} \phi_{i j}\left(\left(\tilde{u}_{k}\right)_{i}\left(\tilde{u}_{k}-u\right)_{j}-\left(u-\tilde{u}_{k}\right)_{i} u_{j}\right)\right\|_{0, \partial \Omega}+\|\rho \mathscr{F}\|_{0} .
$$

By (5.6),

$$
\begin{aligned}
\|\mathscr{F}\|_{0} & \leq\left\|(u \cdot \nabla)\left(\tilde{u}_{k}-u\right)\right\|_{0}+\left\|\left(\left(\tilde{u}_{k}-u\right) \cdot \nabla\right) \tilde{u}_{k}\right\|_{0} \\
& \leq C_{4}\left\|\tilde{u}_{k}-u\right\|_{1} \leq C_{4}\left(\left\|\tilde{u}^{*}-\tilde{u}_{k}\right\|_{1}+k\right),
\end{aligned}
$$


and

$$
\begin{aligned}
& \left\|\sum_{i, j} \phi_{i j}\left(\left(\tilde{u}_{k}\right)_{i}\left(\tilde{u}_{k}-u\right)_{j}-\left(u-\tilde{u}_{k}\right)_{i} u_{j}\right)\right\|_{0, \partial \Omega} \\
& \quad \leq C_{4}\left\|\tilde{u}_{k}-u\right\|_{0, \partial \Omega} \leq C_{4}\left\|\tilde{u}_{k}-u\right\|_{1, \Omega} \leq C_{4}\left(\left\|\tilde{u}^{*}-\tilde{u}_{k}\right\|_{1}+k\right) .
\end{aligned}
$$

Therefore,

$$
\left[\tilde{p}^{*}-\tilde{p}_{k}\right]_{1} \leq C_{4}\left(\left\|\tilde{u}^{*}-\tilde{u}_{k}\right\|_{1}+k\right) .
$$

Substituting (5.14), (5.15) into (5.13), we obtain

$$
\left\|\left(\tilde{u}^{*}-\tilde{u}_{k}\right)(t)-\left(\tilde{u}^{*}-\tilde{u}_{k}\right)(i k)\right\|_{0} \leq C_{4} k\left(\sup _{[i k,(i+1) k)}\left\|\tilde{u}^{*}-\tilde{u}_{k}\right\|_{1}+k\right) .
$$

Let $U=\nabla \Lambda \Psi$. By definition of the function $\Psi$ we have

$$
\begin{aligned}
\Psi(y)-\psi(y) & =-\int_{i k}^{(i+1) k} \sum_{j} \partial_{j} \psi(\xi(y, t ;(i+1) k)) \frac{d}{d t} \xi_{j}(y, t ;(i+1) k) d t \\
& =-\int_{i k}^{(i+1) k} \sum_{j} \partial_{j} \psi(\xi(y, t ;(i+1) k)) u_{j}(\xi(y, t ;(i+1) k)) d t .
\end{aligned}
$$

Since $u, \partial_{1} u, \partial_{2} u, \partial_{1} \xi, \partial_{2} \xi$ are bounded, we get

$$
\|\nabla \Lambda(\Psi-\psi)\|_{0} \leq C_{0} k[\psi]_{2},
$$

that is

$$
\left\|U-\left(\tilde{u}^{*}-\tilde{u}_{k}\right)(i k)\right\|_{0} \leq C_{0} k\left\|\left(\tilde{u}^{*}-\tilde{u}_{k}\right)(i k)\right\|_{1} .
$$

By (5.16), (5.17) we have

$$
\left\|U-\left(\tilde{u}^{*}-\tilde{u}_{k}\right)((i+1) k-0)\right\|_{0} \leq C_{4} k\left(\sup _{[i k,(i+1) k)}\left\|\tilde{u}^{*}-\tilde{u}_{k}\right\|_{1}+k\right) .
$$

Since $\theta=-\nabla \Lambda U$, by (5.11), (5.18), and (2.6) we have

$$
\left\|U-\left(\tilde{u}^{*}-\tilde{u}_{k}\right)((i+1) k-0)\right\|_{1} \leq C_{4} k\left(\sup _{i k \leq \tau<(i+1) k}\left\|\tilde{u}^{*}(\tau)-\tilde{u}_{k}(\tau)\right\|_{1}+k\right) .
$$

But we know that $U \in\left(H_{0}^{1}(\Omega)\right)^{2}, \nabla \cdot U=0$, hence $(I-\Theta) U=0$. $\Theta$ is a bounded operator, thus

$$
\begin{aligned}
\left\|(I-\Theta)\left(\tilde{u}^{*}-\tilde{u}_{k}\right)((i+1) k-0)\right\|_{1} \\
\quad=\left\|(I-\Theta)\left(U-\left(\tilde{u}^{*}-\tilde{u}_{k}\right)((i+1) k-0)\right)\right\|_{1} \\
\quad \leq C_{4} k\left(\sup _{i k \leq \tau<(i+1) k}\left\|\tilde{u}^{*}(\tau)-\tilde{u}_{k}(\tau)\right\|_{1}+k\right) .
\end{aligned}
$$

Lemma 10. If $1 \leq s<\frac{3}{2}, k \leq 1,\left\|\tilde{u}_{k}(t)\right\|_{s+1} \leq M_{2}$ for $i k \leq t<(i+1) k$, then

$$
\left\|u_{k}(t)\right\|_{3} \leq C_{5}(t-i k)^{s / 2-1}
$$


on the same interval, where the constant $C_{5}$ depends only on the domain $\Omega$, the operator $\Theta$, the constants $s, \nu, T, M_{2}$, the functions $f, u_{0}$, and the solution $u$ of $(1.1)-(1.4)$.

Proof. Let $w=\partial u_{k} / \partial t, \pi=\partial p_{k} / \partial t$. Differentiating equations (1.10)-(1.12) formally with respect to $t$, we obtain

$$
\begin{aligned}
\frac{\partial w}{\partial t}+\frac{1}{\rho} \nabla \pi=\nu \Delta w & \quad \nabla \cdot w=0,\left.\quad w\right|_{x \in \partial \Omega}=0 \\
w(i k)=\left.\frac{\partial u_{k}}{\partial t}\right|_{t=i k}= & -\nu(A-I) \Theta \tilde{u}_{k}((i+1) k-0) \\
& +\frac{1}{k} P(I-\Theta) \tilde{u}_{k}((i+1) k-0) .
\end{aligned}
$$

It was proved in $[9$, Chapter $4, \S 2$, Corollary 1$]$ that $\partial u_{k} / \partial t$ is the weak solution of it. But the above problem possesses a strong solution

$$
w(t)=e^{-\nu(t-i k)(A-I)} w(i k)
$$

therefore,

$$
\frac{\partial u_{k}}{\partial t}=e^{-\nu(t-i k)(A-I)} w(i k)
$$

By (2.13), (2.14),

$$
\begin{aligned}
\left\|\frac{\partial u_{k}}{\partial t}\right\|_{1} & \leq C\left\|A^{1 / 2} e^{-\nu(t-i k)(A-I)} w(i k)\right\|_{0} \\
& =C\left\|A^{1-s / 2} e^{-\nu(t-i k)(A-I)} A^{(s-1) / 2} w(i k)\right\|_{0} \\
& \leq C(t-i k)^{s / 2-1}\left\|A^{(s-1) / 2} e^{\nu(t-i k)} w(i k)\right\|_{0} \\
& \leq C(t-i k)^{s / 2-1}\|w(i k)\|_{s-1} .
\end{aligned}
$$

Applying the operator $P$ to equation (1.10), we obtain

$$
\frac{\partial u_{k}}{\partial t}=-\nu(A-I) u_{k}+\frac{1}{k} P(I-\Theta) \tilde{u}_{k}((i+1) k-0) .
$$

Consequently,

$$
\begin{aligned}
\left\|u_{k}\right\|_{3} & \leq C\left\|A u_{k}\right\|_{1} \\
& =C\left\|u_{k}+\frac{1}{k \nu} P(I-\Theta) \tilde{u}_{k}((i+1) k-0)-\frac{1}{\nu} \frac{\partial u_{k}}{\partial t}\right\|_{1} \\
& =C\left\|u_{k}(i k)+\int_{i k}^{t} \frac{\partial u_{k}}{\partial \tau} d \tau+\frac{1}{k \nu} P(I-\Theta) \tilde{u}_{k}((i+1) k-0)-\frac{1}{\nu} \frac{\partial u_{k}}{\partial t}\right\|_{1} .
\end{aligned}
$$


Then by (5.20),

$$
\begin{aligned}
\left\|u_{k}\right\|_{3} \leq & C\left\|u_{k}(i k)+\frac{1}{k \nu} P(I-\Theta) \tilde{u}_{k}((i+1) k-0)\right\|_{1} \\
& +C(t-i k)^{s / 2-1}\|w(i k)\|_{s-1} \\
\leq & C\left\|u_{k}(i k)\right\|_{1}+\frac{1}{k \nu}\left\|P(I-\Theta) \tilde{u}_{k}((i+1) k-0)\right\|_{1} \\
& +C(t-i k)^{s / 2-1} \|-\nu(A-I) \Theta \tilde{u}_{k}((i+1) k-0) \\
& \quad+\frac{1}{k} P(I-\Theta) \tilde{u}_{k}((i+1) k-0) \|_{s-1}
\end{aligned}
$$

By Lemma 9 and (5.6),

$$
\begin{aligned}
& \frac{1}{k}\left\|P(I-\Theta) \tilde{u}_{k}((i+1) k-0)\right\|_{1} \\
& \leq \frac{1}{k}\left\|P(I-\Theta)\left(\tilde{u}^{*}-\tilde{u}_{k}\right)((i+1) k-0)\right\|_{1} \\
& \quad+\frac{1}{k}\left\|P(I-\Theta)\left(u-\tilde{u}^{*}\right)((i+1) k-0)\right\|_{1} \\
& \leq C_{5}\left(\sup _{i k \leq \tau<(i+1) k}\left\|\tilde{u}^{*}(\tau)\right\|_{1}+\sup _{i k \leq \tau<(i+1) k}\left\|\tilde{u}_{k}(\tau)\right\|_{1}+C_{0}\right) \leq C_{5} .
\end{aligned}
$$

Substituting this into (5.21), we obtain (5.19).

Lemma 11. If $1<s<\frac{3}{2},\left\|\tilde{u}_{k}\right\|_{s+1} \leq M_{3}$, then

$$
\sup _{0 \leq t \leq T}\left(\left\|u(t)-u_{k}(t)\right\|_{1},\left\|u(t)-\tilde{u}_{k}(t)\right\|_{1}\right) \leq C_{6} k
$$

where the constant $C_{6}$ depends only on the domain $\Omega$, the operator $\Theta$, the constants $s, \nu, T, M_{3}$, the functions $f, u_{0}$, and the solution $u$ of (1.1)(1.4).

Proof. We denote by $C_{6}$ a generic constant which possesses the above property. Taking the inner product of $(5.8)$ with $\tilde{\omega}^{*}-\tilde{\omega}_{k}$ and noting that

$$
\left(u \cdot \nabla\left(\tilde{\omega}^{*}-\tilde{\omega}_{k}\right), \tilde{\omega}^{*}-\tilde{\omega}_{k}\right)=0
$$

we obtain

$$
\frac{1}{2} \frac{d}{d t}\left\|\tilde{\omega}^{*}-\tilde{\omega}_{k}\right\|_{0}^{2} \leq\left\|u \cdot \nabla\left(\tilde{\omega}^{*}-\omega\right)-\left(u-\tilde{u}_{k}\right) \cdot \nabla \tilde{\omega}_{k}\right\|_{0}\left\|\tilde{\omega}^{*}-\tilde{\omega}_{k}\right\|_{0} .
$$

By (5.10), the right-hand side is bounded by

By (5.12), (5.14) we get

$$
\frac{C_{6}}{2}\left(\left\|\tilde{u}^{*}-\tilde{u}_{k}\right\|_{1}^{2}+k^{2}\right)+\frac{1}{2}\left\|\tilde{\omega}^{*}-\tilde{\omega}_{k}\right\|_{0}^{2} .
$$

$$
\begin{aligned}
\frac{1}{2} \frac{d}{d t}\left\|\tilde{u}^{*}-\tilde{u}_{k}\right\|_{0}^{2} & \leq \frac{1}{2}\left(\|\mathscr{F}\|_{0}^{2}+\left\|\tilde{u}^{*}-\tilde{u}_{k}\right\|_{0}^{2}\right) \\
& \leq C_{6}\left(\left\|\tilde{u}^{*}-\tilde{u}_{k}\right\|_{1}^{2}+k^{2}\right)+\frac{1}{2}\left\|\tilde{u}^{*}-\tilde{u}_{k}\right\|_{0}^{2} .
\end{aligned}
$$


Thus we have

$$
\frac{d}{d t}\left[\tilde{\omega}^{*}-\tilde{\omega}_{k}\right]_{0}^{2} \leq C_{6}\left(\left[\tilde{\omega}^{*}-\tilde{\omega}_{k}\right]_{0}^{2}+k^{2}\right)
$$

By the Gronwall lemma,

$$
\left[\left(\tilde{\omega}^{*}-\tilde{\omega}_{k}\right)(t)\right]_{0}^{2} \leq e^{C_{6} k}\left(\left[\left(\tilde{\omega}^{*}-\tilde{\omega}_{k}\right)(i k)\right]_{0}^{2}+C_{6} k^{3 m}\right) .
$$

Using the triangle inequality,

$$
\begin{aligned}
{\left[\left(\omega^{*}-\omega_{k}\right)(i k)\right]_{0} \leq } & {\left[\left(\tilde{\omega}^{*}-\tilde{\omega}_{k}\right)((i+1) k-0)\right]_{0} } \\
& +\left[\left(\tilde{\omega}^{*}-\tilde{\omega}_{k}\right)((i+1) k-0)-\left(\omega^{*}-\omega_{k}\right)(i k)\right]_{0} \\
\leq & {\left[\left(\tilde{\omega}^{*}-\tilde{\omega}_{k}\right)((i+1) k-0)\right]_{0} } \\
& +\left\|(I-\Theta)\left(\tilde{u}^{*}-\tilde{u}_{k}\right)((i+1) k-0)\right\|_{1},
\end{aligned}
$$

hence, by Lemma 9,

$$
\begin{aligned}
{\left[\left(\omega^{*}-\omega_{k}\right)(i k)\right]_{0} \leq } & {\left[\left(\tilde{\omega}^{*}-\tilde{\omega}_{k}\right)((i+1) k-0)\right]_{0} } \\
& +C_{6} k\left(\sup _{i k \leq \tau<(i+1) k}\left\|\left(\tilde{u}^{*}-\tilde{u}_{\cdot k}\right)(\tau)\right\|_{1}+k\right) .
\end{aligned}
$$

By (5.23),

$$
\left[\left(\omega^{*}-\omega_{k}\right)(i k)\right]_{0} \leq\left[\left(\tilde{\omega}^{*}-\tilde{\omega}_{k}\right)((i+1) k-0)\right]_{0}+C_{6} k\left(\left[\left(\tilde{\omega}^{*}-\tilde{\omega}_{k}\right)(i k)\right]_{0}+k\right) \text {. }
$$

Taking the square of both sides of the above inequality and applying (5.23) again, we get

$$
\begin{aligned}
{\left[\left(\omega^{*}-\right.\right.} & \left.\left.\omega_{k}\right)(i k)\right]_{0}^{2} \\
\leq & {\left[\left(\tilde{\omega}^{*}-\tilde{\omega}_{k}\right)((i+1) k-0)\right]_{0}^{2}+2\left[\left(\tilde{\omega}^{*}-\tilde{\omega}_{k}\right)((i+1) k-0)\right]_{0} } \\
& \cdot C_{6} k\left(\left[\left(\tilde{\omega}^{*}-\tilde{\omega}_{k}\right)(i k)\right]_{0}+k\right)+C_{6} k^{2}\left(\left[\left(\tilde{\omega}^{*}-\tilde{\omega}_{k}\right)(i k)\right]_{0}^{2}+k^{2}\right) \\
\leq & {\left[\left(\tilde{\omega}^{*}-\tilde{\omega}_{k}\right)((i+1) k-0)\right]_{0}^{2}+C_{6} k\left(\left[\left(\tilde{\omega}^{*}-\tilde{\omega}_{k}\right)(i k)\right]_{0}^{2}+k^{2}\right) . }
\end{aligned}
$$

By (1.10)-(1.13), $u^{*}-u_{k}, p^{*}-p_{k}$ is the solution of

$$
\begin{gathered}
\frac{\partial\left(u^{*}-u_{k}\right)}{\partial t}+\frac{1}{\rho} \nabla\left(p^{*}-p_{k}\right) \\
=\nu \Delta\left(u^{*}-u_{k}\right)+\frac{1}{k}(I-\Theta)\left(\tilde{u}^{*}-\tilde{u}_{k}\right)((i+1) k-0), \\
\nabla \cdot\left(u^{*}-u_{k}\right)=0, \\
\left.\left(u^{*}-u_{k}\right)\right|_{x \in \partial \Omega}=0, \\
\left(u^{*}-u_{k}\right)(i k)=\Theta\left(\tilde{u}^{*}-\tilde{u}_{k}\right)((i+1) k-0) .
\end{gathered}
$$

By Lemmas 1 and 2,

$$
\begin{aligned}
\left\|\left(u^{*}-u_{k}\right)(t)\right\|_{0}^{2} \leq e^{k}( & \left\|\left(u^{*}-u_{k}\right)(i k)\right\|_{0}^{2} \\
& \left.\quad+\int_{i k}^{t}\left\|\frac{1}{k}(I-\Theta)\left(\tilde{u}^{*}-\tilde{u}_{k}\right)((i+1) k-0)\right\|_{0}^{2} d \tau\right),
\end{aligned}
$$


Using Lemma 9,

$$
\begin{aligned}
& \left\|\left(\omega^{*}-\omega_{k}\right)(t)\right\|_{0}^{2} \leq\left\|\left(\omega^{*}-\omega_{k}\right)(i k)\right\|_{0}^{2} \\
& \quad+\frac{1}{2 \nu} \int_{i k}^{t}\left\|\frac{1}{k}(I-\Theta)\left(\tilde{u}^{*}-\tilde{u}_{k}\right)((i+1) k-0)\right\|_{0}^{2} d \tau .
\end{aligned}
$$

$$
\begin{aligned}
{\left[\left(\omega^{*}-\omega_{k}\right)(t)\right]_{0}^{2} \leq e^{k}(} & {\left[\left(\omega^{*}-\omega_{k}\right)(i k)\right]_{0}^{2} } \\
& \left.+C_{6} k\left(\sup _{i k \leq \tau<(i+1) k}\left\|\left(\tilde{u}^{*}-\tilde{u}_{k}\right)(\tau)\right\|_{1}^{2}+k^{2}\right)\right) .
\end{aligned}
$$

By (5.23),

$$
\left[\left(\omega^{*}-\omega_{k}\right)(t)\right]_{0}^{2} \leq e^{k}\left(\left[\left(\omega^{*}-\omega_{k}\right)(i k)\right]_{0}^{2}+C_{6} k\left(\left[\left(\tilde{\omega}^{*}-\tilde{\omega}_{k}\right)(i k)\right]_{0}^{2}+k^{2}\right)\right) .
$$

By (5.26), (5.24), (5.23) we obtain

$$
\left[\left(\omega^{*}-\omega_{k}\right)((i+1) k-0)\right]_{0}^{2} \leq\left(1+C_{6} k\right)\left[\left(\tilde{\omega}^{*}-\tilde{\omega}_{k}\right)(i k)\right]_{0}^{2}+C_{6} k^{3} .
$$

Using the initial condition (1.9),

$$
\left[\left(\omega^{*}-\omega_{k}\right)((i+1) k-0)\right]_{0}^{2} \leq\left(1+C_{6} k\right)\left[\left(\omega^{*}-\omega_{k}\right)(i k-0)\right]_{0}^{2}+C_{6} k^{3} .
$$

Therefore, by induction,

$$
\left[\left(\omega^{*}-\omega_{k}\right)((i+1) k-0)\right]_{0}^{2} \leq C_{6} k^{2} e^{C_{6} T},
$$

hence

$$
\left\|\left(u^{*}-u_{k}\right)((i+1) k-0)\right\|_{1}^{2} \leq C_{6} k^{2} .
$$

Applying (5.23), (5.24), (5.26), and (5.6), we obtain (5.22).

Lemma 12. If $i \geq 0,0 \leq s<\frac{3}{2}$, and $\left\|\tilde{u}_{k}(t)\right\|_{s+1} \leq M_{2}$ for $i k \leq t<(i+1) k$, then $\left\|u_{k}(t)\right\|_{s+1} \leq M_{4}$ on the same interval, where the constant $M_{4}$ depends only on the domain $\Omega$, the operator $\Theta$, the constants $\nu, s, T, M_{2}$, the functions $f, u_{0}$, and the solution $u$ of problem (1.1)-(1.4).

Proof. We apply Lemma 3 to the initial-boundary value problem (5.25) and obtain

$$
\begin{aligned}
\left\|\left(u^{*}-u_{k}\right)(t)\right\|_{s+1} \leq C( & \left\|\Theta\left(\tilde{u}^{*}-\tilde{u}_{k}\right)((i+1) k-0)\right\|_{s+1} \\
& \left.+\frac{1}{k}\left\|(I-\Theta)\left(\tilde{u}^{*}-\tilde{u}_{k}\right)((i+1) k-0)\right\|_{1}\right) .
\end{aligned}
$$

It is known that $\left\|\tilde{u}^{*}\right\|_{s+1}$ is bounded, and by Lemma 9 we can estimate the right-hand side. The upper bound of $\left\|\left(u^{*}-u_{k}\right)(t)\right\|_{s+1}$ is given, and $\left\|u^{*}\right\|_{s+1}$ is also bounded; thus the desired upper bound of $\left\|u_{k}(t)\right\|_{s+1}$ follows.

\section{Proof of the Theorem}

We assume that $1<s<\frac{3}{2}$. Let $M_{0}=2 \max _{0 \leq t \leq T} \|\left(u(t) \|_{1}\right.$. We take $m=3$ and determine the constant $C_{1}$ in Lemma 6; then we determine the constant 
$M_{2}$ in Lemma 8 and the constant $C_{5}$ in Lemma 10. By Lemma 6, we take $k_{0} \in(0,1]$ such that

$$
k_{0} \leq \frac{1}{C\left(C_{5} k_{0}^{s / 2-1}+\sup _{0 \leq t \leq T}\|f(t)\|_{3}+1\right)},
$$

that is,

$$
C\left(C_{5} k_{0}^{s / 2}+k_{0} \sup _{0 \leq t \leq T}\|f(t)\|_{3}+k_{0}\right) \leq 1,
$$

which always holds if $k_{0}$ is small enough. By (5.4) we set

$$
M_{5}=C_{3}+C M_{0}^{2-1 / s} M_{2}^{1 / s}+M_{0}^{2-q / s} M_{2}^{q / s},
$$

where $1<q<s$. By Lemma 6 we set

$$
M_{3}=\max \left(C_{1} M_{5}+C_{1}, M_{2}\right) .
$$

We determine the constant $C_{6}$ according to Lemma 11 , and the constant $M_{4}$ according to Lemma 12 , and reduce $k_{0}$, if necessary, such that

$$
\begin{gathered}
\left\|u_{0}\right\|_{3} \leq C_{5} k_{0}^{s / 2-1}, \\
C_{6} k_{0} \leq M_{0} / 2 .
\end{gathered}
$$

With the constants so determined, we prove by induction that if $0<k \leq k_{0}$, then

$$
\begin{gathered}
\left\|\tilde{u}_{k}(t)\right\|_{1} \leq M_{0}, \quad\left\|u_{k}(t)\right\|_{1} \leq M_{0}, \quad\left\|\tilde{u}_{k}(t)\right\|_{s+1} \leq M_{2}, \\
\left\|u(t)-u_{k}(t)\right\|_{1} \leq C_{6} k, \quad\left\|u(t)-\tilde{u}_{k}(t)\right\|_{1} \leq C_{6} k .
\end{gathered}
$$

Two cases are considered simultaneously: (a) $j=0$; (b) $j>0$ and the above assertion is valid for $0 \leq t<j k$. If $j>0$, then by (5.4) and (6.2),

$$
\left\|u_{k}(j k-0)\right\|_{s+1} \leq M_{5} \text {. }
$$

(6.6) also holds for $j=0$. If $j>0$, then by Lemma 10 ,

$$
\left\|u_{k}(j k-0)\right\|_{3} \leq C_{5} k^{s / 2-1}
$$

by (6.4) this also holds for $j=0$. Using Lemma 6 and (6.1), (6.3), $\left\|\tilde{u}_{k}(t)\right\|_{s+1} \leq$ $M_{3}$ for $j k \leq t<(j+1) k$. By Lemma 11 ,

$$
\left\|u(t)-u_{k}(t)\right\|_{1},\left\|u(t)-\tilde{u}_{k}(t)\right\|_{1} \leq C_{6} k
$$

always holds for $0 \leq t<(j+1) k$; by $(6.5),\left\|\tilde{u}_{k}(t)\right\|_{1} \leq M_{0},\left\|u_{k}(t)\right\|_{1} \leq M_{0}$ on the same interval. By Lemmas 6 and $8,\left\|\tilde{u}_{k}(t)\right\|_{s+1} \leq M_{2}$ for $0 \leq t<(j+1) k$. Thus the induction is complete.

Applying Lemma 12, we obtain the upper bound of $\left\|u_{k}(t)\right\|_{s+1}$.

\section{REMARK}

If the Euler equation possesses global solutions, then the conclusion of the theorem is also true for $k \geq k_{0}$; since there are at most $1+\left[T / k_{0}\right]$ steps, the upper bounds in (1.14) and (1.15) are easily obtained. 
A sufficient condition for global existence was given in [8, 11], namely the initial value $u_{0}$ and body force $f$ should satisfy, in addition, $\nabla \Lambda u_{0} \in L^{1}(\Omega)$, $\nabla \Lambda f \in L^{1}(\Omega \times(0, T))$. Under that restriction we can prove by induction global existence for problem (1.6)-(1.9), for any $i$. In fact, if $\omega_{k}(i k-0) \in L^{1}$, then $\tilde{\omega}_{k}(t) \in L^{1}(\Omega)$ for $t \in[i k,(i+1) k)$ (see [11]). For the operator $\Theta$ given in $\S 2$, $(I-\Theta) \tilde{u}_{k}((i+1) k-0)$ has compact support, so $\omega_{k}(i k)=-\nabla \Lambda \Theta \tilde{u}_{k}((i+1) k-0) \epsilon$ $L^{1}(\Omega)$, and $\omega_{k}$ satisfies

$$
\begin{gathered}
\frac{\partial \omega_{k}}{\partial t}=\nu \Delta \omega_{k}-\nabla \Lambda(I-\Theta) \tilde{u}_{k}((i+1) k-0), \\
\left.\omega\right|_{t=i k}=\omega_{k}(i k) .
\end{gathered}
$$

Using the fundamental solution of the heat equation, it is easy to prove that $\omega_{k}(t) \in L^{1}(\Omega)$ for $t \in[i k,(i+1) k)$.

\section{ACKNOWLEDGMENT}

This paper was completed while the author was a visiting professor at the Department of Mathematics, Ohio University. The author is grateful for the hospitality of the Department and of Professor S. Wen. The author also thanks the referee for bringing to his attention the work of G. Benfatto and M. Pulvirenti [5].

\section{BIBLIOGRAPHY}

1. R. A. Adams, Sobolev spaces, Academic Press, New York, 1975.

2. S. Agmon, A. Douglis, and L. Nirenberg, Estimates near the boundary for solutions of elliptic partial differential equations satisfying general boundary conditions. I, Comm. Pure Appl. Math. 17 (1959), 623-727.

3. G. Alessandrini, A. Douglis, and E. Fabes, An approximate layering method for the NavierStokes equations in bounded cylinders, Ann. Mat. Pura Appl. 135 (1983), 329-347.

4. J. T. Beale and A. Majda, Rate of convergence for viscous splitting of the Navier-Stokes equations, Math. Comp. 37 (1981), 243-259.

5. G. Benfatto and M. Pulvirenti, Convergence of Chorin-Marsden product formula in the halfplane, Comm. Math. Phys. 106 (1986), 427-458.

6. A. J. Chorin, Numerical study of slightly viscous flow, J. Fluid Mech. 57 (1973), 785-796.

7. H. Fujita and T. Kato, On the Navier-Stokes initial value problem. I, Arch. Rational Mech. Anal. 16 (1964), 269-315.

8. T. Kato, On classical solutions of the two-dimensional non-stationary Euler equation, Arch. Rational Mech. Anal. 25 (1967), 188-200.

9. O. A. Ladyzhenskaya, The mathematical theory of viscous incompressible flow, Gordon and Breach, New York, 1969.

10. J. L. Lions and E. Magenes, Nonhomogeneous boundary value problems and applications, Springer-Verlag, 1972.

11. F. J. McGrath, Nonstationary plane flow of viscous and ideal fluids, Arch. Rational Mech. Anal. 27 (1968), 329-348.

12. R. Temam, On the Euler equations of incompressible perfect fluids, J. Funct. Anal. 20 (1975). 32-43.

13. L.-a. Ying, Viscosity splitting method in hounded domains, Sci. Sinica Ser. A 32 (1989). 908-921. 
14. L.-a. Ying, The viscosity splitting method for the Navier-Stokes equations in bounded domains, Science Report, Department of Mathematics and Institute of Mathematics, Peking University, October 1986.

15. __ On the viscosity splitting method for initial boundary value problems of the NavierStokes equations, Chinese Ann. Math. 10B (1989), 487-512.

16. __ Viscosity splitting method for three dimensional Navier-Stokes equations, Acta Math. Sinica (N.S.) 4 (1988), 210-226.

17. __ Convergence study of viscous splitting in bounded domains, Lecture Notes in Math., vol. 1297, Springer-Verlag, 1987, pp. 184-202.

Department of Mathematics, Peking University, Beijing, 100871, China 